\title{
A Novel Hybrid Dual Analyzer SIMS Instrument for Improved Surface and 3D- Analysis
}

Alexander Pirkl ${ }^{1}$, Rudolf Moellers ${ }^{1}$, Henrik Arlinghaus ${ }^{1}$, Felix Kollmer ${ }^{1}$, Ewald Niehuis ${ }^{1}$, Alexander Makarov $^{2}$, Stevan Horning ${ }^{2}$, Melissa Passarelli ${ }^{3}$, Rasmus Havelund ${ }^{3}$, Paulina Rakowska ${ }^{3}$, Alan Race ${ }^{3}$, Alexander G. Shard ${ }^{3}$, Andy West ${ }^{4}$, Peter Marshall ${ }^{4}$, Carla F. Newman ${ }^{4}$, Morgan Alexander ${ }^{5}$, Colin Dollery ${ }^{4}$, Ian S. Gilmore ${ }^{3}$

1. ION-TOF GmbH, Muenster, Germany.

2. Thermo Fisher Scientific, Bremen, Germany.

3. National Physical Laboratory, Teddington, United Kingdom.

4. GlaxoSmithKline, Stevenage, United Kingdom.

5. University of Nottingham, Nottingham, United Kingdom.

Time-of-flight secondary ion mass spectrometry (TOF-SIMS) is an established, highly sensitive analytical technique for mass spectrometry (MS) imaging applications with a lateral resolution below $100 \mathrm{~nm}$. Elemental and molecular information is obtained by bombarding the surface with a focused primary ion beam and analyzing the generated secondary ions in a TOF mass analyzer. Furthermore 3D imaging is possible by employing a lower energetic quasi DC sputter beam for material removal (sputter cycle) and a short pulsed small spot analysis beam for optimal mass spectral and imaging performance (so-called dual beam mode). Application of this technique for the localization of drugs and their metabolites in drug-doped cells could be used to find regions in which a pharmaceutical compound accumulates. This would be extremely helpful for selection of possible drug candidates in pre-clinical studies, thereby reducing the development costs for new pharmaceutical products. Furthermore surveying biologically relevant molecules, like lipids, in tissue can give valuable information on the molecular fundamentals of diseases and the effects of treatments.

However in complex biological samples identification of unknown compounds can be hampered by mass interferences and a high number of possible assignments for a single mass peak. Additionally ions generated in the sputter cycle of a dual beam TOF-SIMS experiment cannot be utilized for the analysis, because the long pulses and high primary ion currents in the sputter phase prohibit the use of a TOF analyzer. In order to overcome these limitations, the 3D nanoSIMS project [1] is developing a revolutionary new SIMS instrument that combines the high lateral resolution and speed associated with TOF-SIMS with the high mass resolution and high mass accuracy of an orbital trapping mass analyzer.

The prototype instrument combines a dedicated, fast TOF analyzer (TOF.SIMS 5, ION-TOF GmbH, Muenster, Germany) with an orbital trapping analyzer (QExactive ${ }^{\mathrm{TM}}$ HF [2], Thermo Fisher Scientific, Bremen, Germany). The hybrid design allows alternating analysis of samples with either short pulsed analysis beams and the TOF analyzer or quasi DC pulses and the Orbitrap ${ }^{\mathrm{TM}}$. The prototype is equipped with two newly developed ion sources: 1.) a gas cluster ion beam column allowing a lateral resolution down to the micron level with minimal sub-surface damage and 2.) a liquid metal ion gun for highest lateral resolution below $80 \mathrm{~nm}$, featuring a DC mass filter for generation of very long ion pulses of welldefined metal ion species.

In this paper we demonstrate the analytical performance of this instrument for the analysis of biological samples. To show the potential of drug analysis in cells Amiodarone-dosed NR8383 cells were analyzed 
using a bismuth cluster primary ion beam. The high lateral resolution TOF-SIMS image in Fig. 1 shows the sub-cellular distribution of iodine, a moiety of the drug amiodarone (green) with respect to the nucleus of the cell (red). In gas cluster SIMS spectra with the Orbitrap analyzer the signal of the intact drug molecule can be detected with a mass accuracy below 3 ppm and a mass resolution of above 140000 .

First gas cluster SIMS images using the Orbitrap analyzer from coronal mouse brain sections demonstrate simultaneous localization and identification of various biological compounds. On tissue, the high mass resolution (FWHM of 240000 at m/z 200, >100 000 in the lipid mass region) was used to separate and identify lipids and metabolites with sub-ppm mass accuracy. In many cases, the mass spectra exhibited multiple molecular ion signatures at the same nominal mass unit in the lipid region. For example two almost isobaric peaks m/z 904.62 and m/z 904.66 were fully resolved and displayed different localization. These peaks were identified as C24:1 (OH) sulfatide [(3'-sulfo)GalCer(d18:1/24:1(2OH))] and C25:0 sulfatide [(3'-sulfo)Gal-Cer(d18:1/25:0)] with a mass accuracy of 1.1 and $1.0 \mathrm{ppm}$, respectively. Furthermore over 50 glycosphingolipid sulfatide species were identified and mapped. Both hydroxylated and non-hydroxylated sulfatides with chain length ranging from 14 to 26 and varying degrees of fatty acid saturation were identified. The presence and assignment of $\mathrm{C} 24(\mathrm{OH})$ Sulfatide [(3'-sulfo)Gal $\beta$-Cer(d18:1/24:0(2OH))] was confirmed with in situ tandem MS spectra of the suspected sulfatide peak at $\mathrm{m} / \mathrm{z} 906$.

\section{References:}

[1] The 3D nanoSIMS project, http://www.npl.co.uk/news/3d-nanosims-label-free-molecular-imaging

[2] Scheltema, et al. Mol Cell Proteomics (2014).

[3] Passarelli, et al. Anal. Chem. (2015).
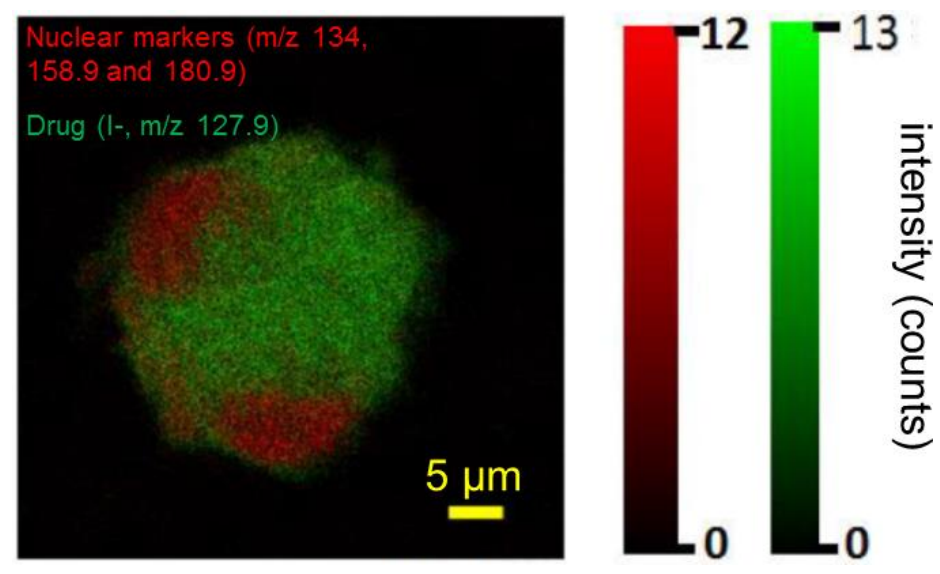

Figure 1. TOF-SIMS image showing the uptake of the drug amiodarone (green) into a single macrophage [3]. 